11.4

\title{
Эксперимент по определению времени прохождения сверхширокополосных хаотических радиоимпульсов через многолучевой канал
}

\author{
(C) Л.В. Кузьмин, Е.В. Ефрремова
}

Институт радиотехники и электроники им. В.А. Котельникова РАН, Москва, Россия

E-mail: Ivkuzmin@gmail.com

Поступило в Редакцию 23 апреля 2020г.

В окончательной редакции 23 апреля 2020г.

Принято к публикации 14 мая 2020г.

\begin{abstract}
Описываются результаты эксперимента по определению времени распространения сверхширокополосных хаотических радиоимпульсов через беспроводный канал с многолучевым распространением в диапазоне частот от 3 до $5 \mathrm{GHz}$. Проведена оценка точности измерения времени распространения сверхширокополосных хаотических радиоимпульсов по их огибающей для решения задачи измерения расстояния между объектами и их локализации в пространстве в промышленных и офисных помещениях.
\end{abstract}

Ключевые слова: сверхширокополосные сигналы, хаотические сигналы, хаотические радиоимпульсы, многолучевое распространение, многолучевое замирание, локализация, измерение времени распространения.

DOI: 10.21883/PJTF.2020.16.49849.18352

Измерение времени распространения радиосигналов через беспроводные каналы является актуальной научно-практической задачей. Одна из причин - развитие робототехнических комплексов, в которых требуется осуществлять позиционирование объектов при помощи радиосистем ненаправленного действия в нелицензируемом частотном диапазоне от 2 до $10 \mathrm{GHz}$ (сантиметровый диапазон длин волн). Такие системы предназначены для применения в условиях промышленной, жилой и городской инфраструктуры, где сильны эффекты многолучевого распространения, оказывающие значительное влияние на точность, поэтому вопрос о точности измерения времени распространения радиосигнала в многолучевых каналах стоит очень остро $[1,2]$. Помимо традиционных узкополосных технологий [1] (WiFi, Bluetooth, ZigBee, где расстояние определяется через мощность принимаемого сигнала или направление на излучатель) хорошими кандидатами для решения данной задачи являются шумоподобные и сверхширокополосные (СШП) сигналы, имеющие два полезных фундаментальных свойства: малое время когерентности и большую базу сигнала. Благодаря этому потенциально в приемнике можно реализовать большое накопление и получить высокую точность оценивания параметров сигнала (мощность или фаза) после распространения через канал.

Физико-технические способы оценивания параметров сигналов в конечном итоге определяют возможности конкретных беспроводных технологий. В узкополосных системах точность определения местоположения не превышает $1 \mathrm{~m}[1,2]$. Попытка улучшения точности за счет использования СШП-сигналов сталкивается с многолучевым распространением, которое проявляется в искажении формы ультракоротких (УК) импульсов, их амплитуды и фазы, что ограничивает потенциально очень высокую точность определения момента прихода УК-импульсов ( 100 ps). В СШП-системах, где используются УК-импульсы, например в СШП-модулях Decawave DWM-1000 или Ubisense [2], точность в помещениях не превышает $10 \mathrm{~cm}$ на расстояниях до $10 \mathrm{~m} \mathrm{[3-5].}$

Хаотические СШП-радиоимпульсы при решении этой проблемы свободны от недостатков ультракоротких СШП-импульсов в многолучевых каналах. Огибающая хаотических СШП-радиоимпульсов слабо меняется при прохождении через многолучевой канал, так как это шумоподобный сигнал [6,7], а их длительность может быть выбрана много большей, чем длительность многолучевого отклика канала. Это позволяет осуществить измерение времени распространения сигнала за счет измерения параметров огибающей, а не несущего колебания.

Цель настоящей работы - экспериментально подтвердить возможность измерения времени и расстояния при помощи хаотических СШП-радиоимпульсов СВЧ-диапазона в реальных многолучевых каналах по их огибающей и предложить способ такого измерения.

Эксперимент проводился в лабораторном помещении с использованием опытных образцов СШП-приемопередатчиков [8] в соответствии со схемой, представленной на рис. $1, a$. В излучателе $T_{X}$ под действием модулирующего сигнала $m(t)$ генератор $G_{C}$ хаотических колебаний формировал [9] последовательность $s(t)$ из хаотических радиоимпульсов с длительностью $T_{P}=166 \mathrm{~ns}$, мощностью $P_{P}=50 \mathrm{~mW}$ и периодом следования $2 T_{P}$. После прохождения канала $C$ последовательность $s_{C}(t)$ поступала на вход приемника $E_{D}$, формирующего огибающую $e(t)$ сигнала $s_{C}(t)$. Расстояние между излучателем и приемником менялось в пределах от 25 до $150 \mathrm{~cm}$. Запоминающий осциллограф $(O s c)$ фиксировал реализации 

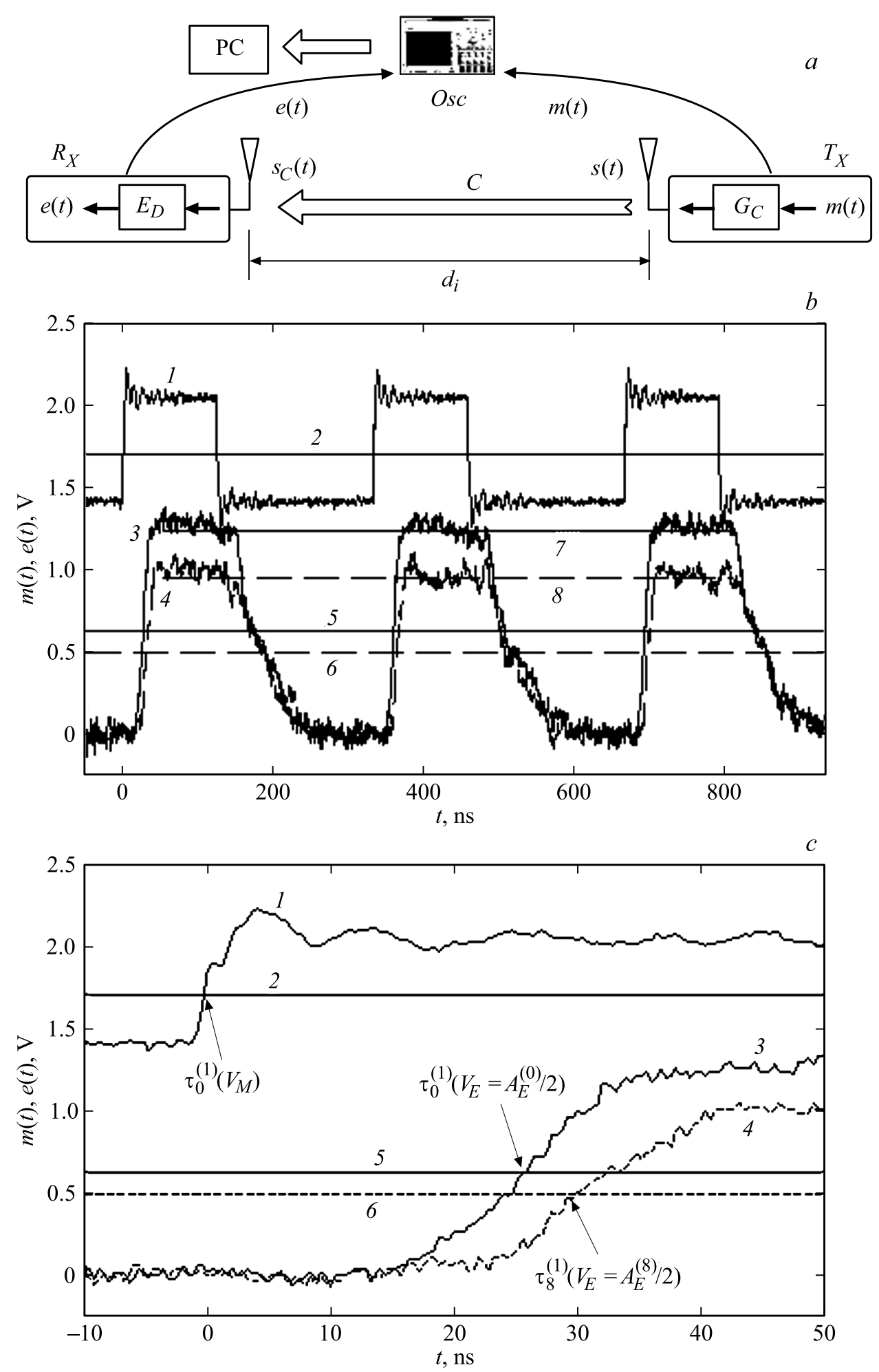

Рис. 1. Схема эксперимента $(a)$ по измерению времени распространения хаотических СШП-радиоимпульсов от излучателя $T_{X}$ до приемника $R_{X}$ на расстояние $d_{i}$ (от $d_{0}=25 \mathrm{~cm}$ до $\left.d_{8}=150 \mathrm{~cm}\right): m(t)-$ сигнал, модулирующий генератор хаотических колебаний $G_{C} ; e(t)$ - сигнал на выходе детектора огибающей импульсов $E_{D} ; C-$ среда распространения (канал). Эпюры $(b$ и $c)$ сигналов в передатчике и приемнике: кривая $1-m(t)$, кривые 3 и $4-e(t)$ для $d_{0}=25 \mathrm{~cm}$ и $d_{8}=150 \mathrm{~cm}$ соответственно; прямая $2-$ пороговое значение напряжения, по которому фиксируется момент излучения радиоимпульсов; прямые 5 и $6-$ пороговые значения напряжений, по которым фиксируется приход импульсов в приемнике для $d_{0}=25 \mathrm{~cm}$ и $d_{8}=150 \mathrm{~cm}$ соответственно; прямые 7 и 8 - среднее значение амплитуды сигнала-огибающей хаотических радиоимпульсов на расстояниях $d_{0}=25 \mathrm{~cm}$ и $d_{8}=150 \mathrm{~cm}$ соответственно. 
сигналов $m(t)$ и $e(t)$ в момент поступления переднего фронта первого импульса последовательности $m(t)$.

Время прохождения хаотических радиоимпульсов измерялось путем сравнения реализаций сигналов $m(t)$ и $e(t)$ (рис. $1, b)$ через вычисление разностей между моментами времени начала импульсов (их передних фронтов) сигнала передатчика $m(t)$ и импульсов сигнала-огибающей $e(t)$ приемника для каждой соответствующей пары импульсов сигналов $m(t)$ и $e(t)$. Данный способ с технической точки зрения является наиболее привлекательным и постоянно обсуждается в литературе [10-13].

Модулирующий сигнал $m(t)$ управляет питанием транзисторного генератора $G_{C}$ хаотических СШП-колебаний, который повторяет начальные фрагменты хаотических радиоимпульсов от импульса к импульсу [9]. Поэтому в приемнике передний фронт огибающей у всех импульсов при одних и тех же условиях распространения с точностью до влияния шума также будет идентичен.

Приемник реализован на базе логарифмического СШП-детектора [14] и малошумящего усилителя с коэффициентом усиления $10^{2}$ и обеспечивает чувствительность на уровне $3 \cdot 10^{-9} \mathrm{~mW}$. На выходе приемника мгновенное значение амплитуды сигнала-огибающей $e(d, t)$ пропорционально логарифму мгновенного значения мощности хаотических СШП-радиоимпульсов на его входе. Амплитуда в линейной зоне работы приемника меняется по закону $e(d, t)=10 \alpha \lg \left(\frac{P(d, t)}{P_{0}}\right)$, где $P_{0}=1 \mathrm{~mW}$ - референтное значение мощности, $\alpha-$ крутизна характеристики логарифмического детектора, $P(d, t)$ - мощность сигнала, поступающая в приемник от излучателя, находящегося на расстоянии $d$ от него.

Детектор обеспечивает изменение амплитуды выходного сигнала $e(d, t)$ от 0.5 до $2 \mathrm{~V}$ при изменении мощности входного сигнала в диапазоне от $3 \cdot 10^{-9}$ до $\sim 10 \mathrm{~mW}$. Этого достаточно, чтобы иметь в эксперименте техническую возможность приема хаотических СШП-сигналов, мощность которых может изменяться от $50 \mathrm{~mW}$ в момент излучения передатчиком до $\sim 0.05 \mathrm{~mW}(25 \mathrm{~cm})$ и до $\sim 0.0025 \mathrm{~mW}(150 \mathrm{~cm})$ в момент поступления в приемник после канала. Это значение мощности задает такое отношение сигнал-шум в приемнике, при котором негативным влиянием шума на точность можно пренебречь $[10-13,15]$ и принимать во внимание результат только многолучевого распространения.

Начало каждого импульса в передатчике фиксировалось по переднему фронту (рис. $1, b)$ в момент $\tau_{M}$ превышения сигналом модулятора $m(t)$ порогового значения напряжения $V_{M}$, а в приемнике - в момент $\tau_{E}$ превышения сигналом $e(t)$ значения $V_{E}$, когда $m\left(\tau_{M}\right)=V_{M}$ и когда $e\left(\tau_{E}\right)=V_{E}$. Разность $\tau_{E}-\tau_{M}$ между ними равна времени распространения импульса от момента начала его формирования передатчиком до момента его появления на выходе приемника. Эта разность включает время $T_{C}$ прохождения сигналом выходных цепей передатчика, входных цепей приемника и время $T_{S}$ распространения от передатчика к приемнику: $T_{D}=\tau_{E}-\tau_{M}=T_{S}+T_{C}$.

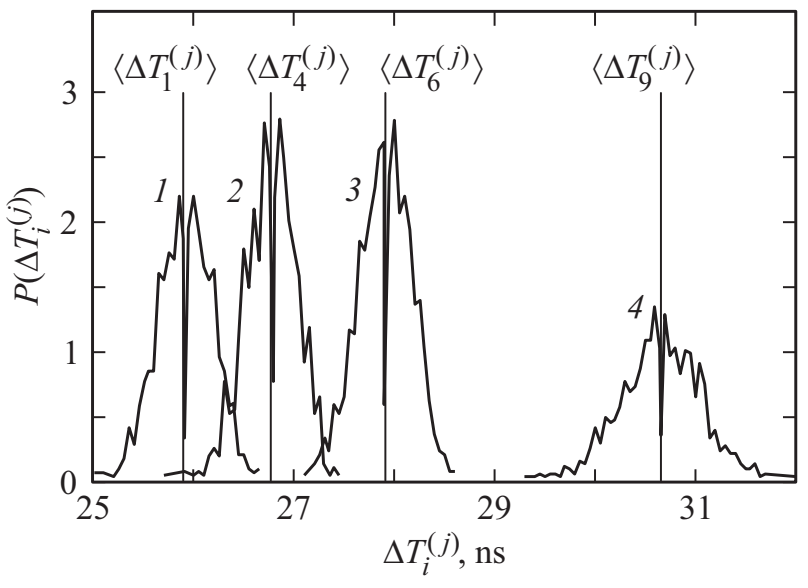

Рис. 2. Распределения времен задержки $P\left(\Delta T_{i}^{(j)}\right)$ прихода сигналов для расстояния $d_{1}(1), d_{4}(2), d_{6}(3)$ и $d_{9}(4)$. Вертикальными линиями обозначены средние значения задержек $\Delta T_{i}=\left\langle\Delta T_{i}^{(j)}\right\rangle$.

В эксперименте были зафиксированы девять реализаций $m_{i}(t)$ и $e_{i}(t), i=0 \ldots 8$, для расстояний $d_{i}=\{25,30,37.5,50,62.5,75,100,125,150\} \mathrm{cm}$ между излучателем и приемником. Реализации $m_{i}(t)$ и $e_{i}(t)$ фиксировались осциллографом с частотой дискретизации $f_{S}=2.5 \mathrm{GHz}$ и разрядностью 12 bit (4096 уровней квантования по амплитуде). Минимальный шаг по времени между отсчетами составляет $0.4 \mathrm{~ns}$, что соответствует расстоянию $\sim 12 \mathrm{~cm}$.

Для определения чистого времени $T_{S}$ прохождения сигналом расстояния через беспроводной канал необходимо исключить неизвестный параметр $T_{C}$. Для этого определялось время $T_{S}\left(\Delta d_{i}\right)$ распространения сигнала на расстояние $\Delta d_{i}=d_{i}-d_{0}$, равное разности

$$
\begin{aligned}
T_{S}\left(\Delta d_{i}\right) & =T_{D}\left(d_{i}\right)-T_{D}\left(d_{0}\right) \\
& =\left(\tau_{E}-\tau_{M}\right)_{i}-\left(\tau_{E}-\tau_{M}\right)_{0}=T_{S}^{(i)}-T_{S}^{(0)}
\end{aligned}
$$

между временами распространения сигнала $T_{D}\left(d_{0}\right)$ для расстояния $d_{0}$ и $T_{D}\left(d_{i}\right)$ для $d_{i}$.

Начальные фрагменты сигналов $e_{i}(t)$ и $m_{i}(t)$ изображены на рис. $1, c$ для расстояний $d_{0}=25 \mathrm{~cm}$ и $d_{8}=150 \mathrm{~cm}$. Передний фронт импульсов нарастает в пределах $\sim 15 \mathrm{~ns}$ (рис. 1,c). С увеличением дистанции между излучателем и приемником амплитуда импульсов уменьшается. Задний фронт импульсов формируется переотраженными сигналами.

Значения $V_{M}$ и $V_{E}$ выбирались равными половине средней амплитуды огибающих импульсов: $V_{M}=V_{M}^{(i)}=\frac{A_{M}^{(i)}}{2}$ и $V_{E}=V_{E}^{(i)}=\frac{A_{E}^{(i)}}{2}$. Это означает, что времена прихода сигналов определяются по моментам, соответствующим одной и той же фазе сигналов. Амплитуда огибающей импульса пропорциональна его мощности, серединное значение соответствует половине от мощности импульса на заданном расстоянии от источника. 

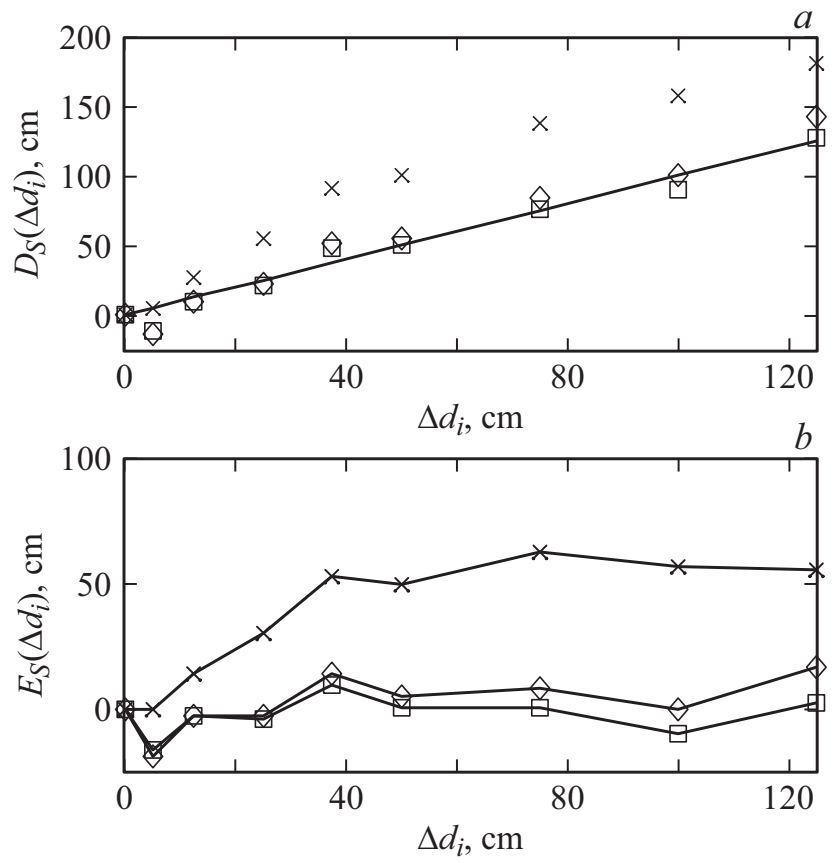

Рис. 3. Зависимости измеренного расстояния $D_{S}\left(\Delta d_{i}\right)(a)$ и средней ошибки $E_{S}\left(\Delta d_{i}\right)$ (b) от истинного $\Delta d_{i}$, определенного путем вычисления средней задержки между излученными и принятыми импульсами (ромбы), усреднения времени прихода импульсов по фиксированному порогу (крестики), минимизации задержки по пачке импульсов (квадраты).

В эксперименте были определены моменты времени $\tau_{i}^{(j)}\left(V_{M}\right)$ начала импульсов сигнала $m(t)$ и моменты $\tau_{i}^{(j)}\left(V_{E}\right)$ начала импульсов сигнала $e(t)$, где $j=1 \ldots 1000$ соответствует номеру импульса в реализациях $m_{i}(t)$ и $e_{i}(t)$. На рис. 2 представлены распределения величин $\Delta T_{i}^{(j)}\left(d_{i}\right)$ измеренного времени прохождения каждого импульса через канал $\Delta T_{i}^{(j)}\left(d_{i}\right)=\tau_{i}^{(j)}\left(V_{E}\right)-\tau_{i}^{(j)}\left(V_{M}\right)$. Вертикальными линиями отмечены положения средних значений $\Delta T_{i}=\left\langle\Delta T_{i}^{(j)}\right\rangle$. Здесь угловые скобки означают усреднение по $j$.

На основании средних значений $\Delta T_{i}$ измеренного времени распространения были вычислены расстояния $D_{S}\left(d_{i}, d_{0}\right)$, соответствующие этому времени (рис. 3, $a): \quad D_{S}\left(d_{i}, d_{0}\right)=c\left(\Delta T_{i}-\Delta T_{0}\right)$, где $c-$ скорость света в вакууме. Сплошная прямая на рис. 3, $a$ соответствует истинному расстоянию. Ошибки измерений $E_{S}\left(\Delta d_{i}\right)=D_{S}\left(d_{i}, d_{0}\right)-\left(d_{i}-d_{0}\right)$ представлены на рис. $3, b$. Величина ошибки меняется от 1 до $15 \mathrm{~cm}$.

Описанный способ определения времени распространения требует знания порогового значения сигнала, зависящего от амплитуды импульса $V_{E}=\frac{A_{E}^{(i)}}{2}$. Практический же интерес представляет измерение времени распространения сигнала путем фиксации момента его прихода по фиксированному порогу $V_{E}=T_{E}$ безотносительно к амплитуде импульсов.

Вычисление моментов времени $\tau_{i}^{(j)}\left(T_{E}=0.5 \mathrm{~V}\right)$ прихода импульсов и соответствующих им расстояний
$D_{S}\left(d_{i}, d_{0}\right)$ для фиксированного порога $V_{E}=T_{E}=0.5 \mathrm{~V}$, не изменяющегося от серии к серии импульсов, показывает, что в этом случае оценка времени и расстояний будет получаться смещенной (рис. 3, $a$, крестики). Этот результат находится в хорошем согласии с данными работы [13], где также обнаружено, что использование фиксированного порога для приема ультракоротких СШП-импульсов энергетическим приемником без поправки на их фазу приводит к смещенным оценкам времени распространения сигнала.

Применение ,пороговой“ техники при условии правильного выбора момента приема сигнала по его фазе решает проблему определения времени распространения хаотических СШП-радиоимпульсов по их огибающей, но при этом возникает вопрос: а нельзя ли измерить время распространения импульсов для пачки в целом, а не для каждого импульса из пачки в отдельности? Далее предлагается такой способ.

Рассмотрим величину $\left(\tau_{i}^{(j)}\left(V_{E}\right)-\Delta\right)-\tau_{i}^{(j)}\left(V_{M}\right)$, где $\Delta$ - параметр. Когда параметр $\Delta$ в точности равен времени распространения импульса, величина $\left(\tau_{i}^{(j)}\left(V_{E}\right)-\Delta\right)-\tau_{i}^{(j)}\left(V_{M}\right)=0$. Если просуммировать по всем импульсам модули этой величины

$$
T(\Delta)=\sum_{j=1}^{N}\left|\left(\tau_{i}^{(j)}\left(V_{E}\right)-\Delta\right)-\tau_{i}^{(j)}\left(V_{M}\right)\right|,
$$

то $T(\Delta)$ будет равна нулю, если для каждого импульса время распространения равно $\Delta$, и отличаться от нуля при наличии ошибок измерения. Будем искать значение $\Delta$, минимизирующее $T(\Delta)$ :

$$
\Delta_{\text {opt }}=\underset{\Delta}{\operatorname{argmin}} \sum_{j=1}^{N}\left|\left(\tau_{i}^{(j)}\left(V_{E}\right)-\Delta\right)-\tau_{i}^{(j)}\left(V_{M}\right)\right| .
$$

Значение $\Delta_{\text {opt }}$ является оценкой времени распространения пачки импульсов. Результаты расчетов приведены на рис. 3, $a$ и $b$. Достигнутая точность оценки времени распространения лучше точности, получаемой при простой ,Пороговой“ технике.

Полученные результаты показывают, что ошибка измерения существенно меньше характерных времен запаздывания лучей в многолучевом канале, которые в условиях проведенного эксперимента в пересчете на расстояние составляли $\sim 1 \mathrm{~m}$.

Результаты подтверждают, что применение хаотических СШП-радиоимпульсов в многолучевом канале позволяет достичь сантиметровой точности при определении расстояния между излучателем и приемником. Эта точность хуже теоретически возможной, но превышает инструментальную, определяемую осциллографом. Описанные способы измерения могут стать основой для осуществления технической реализации предложенных подходов.

\section{Благодарности}

Авторы выражают благодарность за помощь в проведении эксперимента В.В. Ицкову и М.М. Петросяну. 


\section{Финансирование работы}

Работа выполнена в рамках государственного задания Института радиотехники и электроники им. В.А. Котельникова РАН.

\section{Конфликт интересов}

Авторы заявляют, что у них нет конфликта интересов.

\section{Список литературы}

[1] Hölzl M., Neumeier R., Ostermayer G. // Int. J. Distrib. Sensor Networks. 2015. V. 11. N 8. P. 1-11.

[2] Alarifi A., Al-Salman A., Alsaleh M., Alnafessah A., Al-Hadhrami S., Al-Ammar M.A., Al-Khalifa H.S. // Sensors. 2016. V. 16. N 5. P. 707-742.

[3] Poulose A., Eyobu O.S., Kim M., Han D.S. // 11th Int. Conf. on ubiquitous and future networks (ICUFN). Zagreb, Croatia, 2019. P. 84-88.

[4] Wijaya B., Deng N., Jiang K., Yan R., Yang D. // IEEE Int. Vehicles Symp. (IV). Paris, France, 2019. P. 1239-1246.

[5] Schroeer G. // Int. Conf. on indoor positioning and indoor navigation (IPIN). Nantes, France, 2018. P. 1-5.

[6] Кузьмин Л.В., Гриневич А.В. // Письма в ЖТФ. 2019. Т. 45. B. 16. C. 33-36.

[7] Ефремова Е.В., Дмитриев А.С., Кузьмин Л.В. // Письма в ЖТФ. 2019. Т. 45. В. 17. С. 3-7.

[8] Кузьмин Л.В., Рыжсов А.И., Андреев Ю.В., Попов М.Г. // Физические основы приборостроения. 2018. Т. 7. № 1(27). C. 91-102.

[9] Dmitriev A., Efremova E., Kuzmin L., Atanov N. // Int. J. Bifurcat. Chaos. 2007. V. 17. N 10. P. 3443-3448.

[10] D'Andrea A.N., Mengali U., Reggiannini R. // IEEE Trans. Commun. 1994. V. 42. N 234. P. 1391-1399.

[11] Dardari D., Chong C., Win M. // IEEE Trans. Commun. 2008. V. 56. N 8. P. $1366-1378$.

[12] Liu W., Ding H., Huang X., Liu Z. // IEEE Commun. Lett. 2012. V. 16. N 5. P. 738-741.

[13] Zwirello L., Schipper T., Jalilvand M., Zwick T. // IEEE Trans. Instrum. Meas. 2015. V. 64. N 1. P. 39-51.

[14] Analog Devices. Data Sheet. $1 \mathrm{MHz}$ to $4 \mathrm{GHz}, 80 \mathrm{~dB}$ Logarithmic Detector/Controller [Электронный pecypc]. Режим доступа: http://www.analog.com/media/en/technicaldocumentation/data-sheets/ADL5513.pdf

[15] Skolnik M.I. // IRE Trans. Aeronaut. Navig. Electron. 1960. V. ANE-7. N 4. P. 123-129. 